\title{
Lysyl oxidase (LOX) - a future new ally on the stage of the fight against cancer
}

\author{
Alexandra Totan' ${ }^{1}$ Cosmin Totan², Ana Maria Alexandra Stănescu ${ }^{4}$, Daniela Miricescu', \\ Iulia-Ioana Stănescu ${ }^{3}$, Maria Greabu ${ }^{1}$ \\ ${ }^{1}$ Department of Biochemistry, Faculty of Dental Medicine, \\ "Carol Davila" University of Medicine and Pharmacy, Bucharest, Romania \\ 2Department of Oral and Maxillofacial Surgery, Faculty of Dental Medicine, \\ "Carol Davila" University of Medicine and Pharmacy, Bucharest, Romania \\ ${ }^{3}$ Department of Physiology, Faculty of Dental Medicine, \\ "Carol Davila" University of Medicine and Pharmacy, Bucharest, Romania \\ ${ }^{4}$ Department of Family Medicine, "Carol Davila" University of Medicine and Pharmacy, \\ Bucharest, Romania
}

\begin{abstract}
The local extracellular matrix (EM) remodelling is considered one of the critical events in metastasis, both at primary and the secondary sites. One of the main actors on the EM remodelling is lysyl oxidase (LOX). In this connection, the aim of this paper is to bring to light a possible future systemic (and why not, salivary) biomarker of the metastatic process.

LOX best-characterized role is in the EM remodelling by oxidative deamination of collagens and elastin peptidyl lysine residues, in order to facilitate covalent cross-linking.

The LOX family members seem to play extremely important roles in these extracellular matrix interactions. Future research is needed to confirm whether simple LOX blocking or its downstream actions control could be regarded as target of preventive treatment in patients at a higher risk of metastasis. This is how the portrait of the future biomarker of LOX is becoming more and more outlined.
\end{abstract}

Keywords: extracellular matrix, lysyl oxidase, biomarker, metastatic process

\section{INTRODUCTION}

Primary tumors spread to nonadjacent unrelated tissues has been proven to be a complex and very difficult challenge for researchers. This is because metastasis represents a complex systemic process involving the primary tumor cells, but also non-malignant cells at different secondary sites.

It has been shown that cancer cells secrete factors able to modulate the molecular behavior of host cells resident in secondary tissues, in the absence of the originating tumor cells (1). The local extracellular matrix (EM) remodelling is considered one of the critical events in metastasis, both at primary and the secondary sites (1). One of the main actors on the EM remodelling is Lysyl oxidase (LOX) (1). In this connection, the aim of this paper is to bring to light a possible future systemic (and why not, salivary) biomarker of the metastatic process (1-3).

So, let us find out who actually LOX is.

LOX is a member of a five secreted copper-dependent enzymes family, whose main biochemical function is to oxidize primary amines to reactive aldehydes $(4,5)$.

Proteins with LOX activity have been identified not only in animals, but also in other eukaryotes, bacteria and archaea many, highlighting the pre-metazoan origin for this gene family (6).

LOX was first discovered as a result of analysing the effects of Lathyrus oderatus peas consumption. Lathyrus oderatus peas contain a natural LOX inhibitor - $\beta$-aminopripionitrile. Lathyrus oderatus 
peas consumption resulted in men and animals in severe connective tissue abnormalities which were especially apparent in bone (7). $\beta$-Aminopripionitrile is actually a active-site directed irreversible inhibitor of LOX (8). $\beta$-aminopripionitrile is still used in order to define LOX enzymatic activity. LOX inhibition by $\beta$-aminopripionitrile is unique to this enzymes family, while all other tissue-associated and serum amine oxidases remain unaffected or oxidize $\beta$-aminopripionitrile as a substrate (9). So, it can be sustained that an amine oxidase inhibited by low concentrations of $\beta$-aminopripionitrile is, by definition, a LOX, and an amine oxidase resistant to $\beta$-aminopripionitrile is not.

The LOX family includes 2 subgroups (10):

- LOXL1

- LOXL2 - LOXL4; the members of this subgroup have four conserved scavenger receptor cysteine-rich (SRCR) domains in the longer propeptidic region (10).

LOX are expressed in various cell types: basal and suprabasal keratinocytes, fibroblasts, adipocytes, osteoblasts, smooth muscle cells, and endothelial cells (4). Moreover, during fibrosis, LOX mRNA molecules are overexpressed in the oral submucosa, bone marrow, liver, lung, kidney, and skin (11).

\section{LOX BIOSYNTHESIS}

Biosynthesis of LOX enzymes includes the following steps (10):

- secretion of an inactive $50 \mathrm{kDa}$ glycoprotein precursor (Pro-LOX). This precursor contains the copper and quinone cofactors (10);

- Pro-LOX undergoes extracellular proteolytic processing by the procollagen C-proteinases, resulting an active $\sim 30 \mathrm{kDa}$ lysyl oxidase and $\mathrm{a} \sim 18 \mathrm{kDa} \mathrm{N}$ - and O-glycosylated lysyl oxidase propeptide (LOX-PP) (10).

\section{LOX FUNCTIONS}

The essential role played by mammalian LOX was demonstrated using $\mathrm{LOX}^{-/-}$mice, which died just before or soon after birth because of severe cardiovascular malformations, due to defective elastogenesis (12).

LOX best-characterized role is in the EM remodelling by oxidative deamination of collagens and elastin peptidyl lysine residues, in order to facilitate covalent cross-linking $(13,14)$.

Enzyme-dependent collagen cross-linking is based on a constellation of intracellular procolla- gen alpha chains modifications of by lysyl hydroxylases and the extracellular modifications catalysed by LOX. The lysyl hydroxylases are endoplasmic reticulum-associated enzymes depending on $\alpha$-ketoglutarate and ascorbate, cofactors also required by collagen prolyl hydroxylases (15). The lysyl hydroxylases catalyze the hydroxylation of the penultimate carbon atom of specific of procollagen alpha chains lysine residues (15). It is worth noting that only some specific lysine residues are hydroxylated. The hydroxylation degree is tissue-specific (16). Some of these new hydroxyl groups will further become sites of attachment for the collagen carbohydrate moieties of collagen (16). Collagen molecules glycosylation is quite different from the well-known $\mathrm{N}$ - and O-glycosylation pathways, occuring in the case of asparagine and serine/threonine residues of other proteins (16). Collagen glycosylation reactions are catalyzed by hydroxylysyl galactosyltransferase, followed by galactosylhydroxylysyl glucosyltransferase activity in order to produce glucosylgalacosyl hydroxylysine (17).

LOX activity is very important in ensuring the EM structural integrity and stability (13). In this way LOX contributes to the tensile strength of many connective tissues (18). Moreover, LOX can also activate the collagen III and elastin genes promoters, but is able indirectly to represses the cyclin Dlgene promoter $(18,19)$. So, it can be sustained that LOX has a main role in the EM biology, by intra- and extra-cellularly mechanisms.

Also, interesting in vitro studies revealed that LOX is able to oxidize FGF- 2 and TGF- $\beta$, inhibiting in this way their ability to generate signals (20).

It has been shown that LOXL2 played important roles on the angiogenesis stage, in the context of endochondral bone formation and bone healing (11).

In the bone marrow progenitor cells LOXL4 expression may be considered a future, predictive marker of vivo bone formation (21).

Taken all together, the experimental data highlights the fact that LOX biological functions extend beyond collagen cross-linking. Nagan's model regarding LOX activity sustained that this enzyme would bind to the triple helical domain of the procollagen molecule before fibril formation, and then would act on a neighboring collagen molecule telopeptide lysine residue, during fibril formation (22).

The LOX enzyme family is more and more subject of intense research in the cancer context. Very interesting to note is the fact that LOX expression is typically induced under hypoxia conditions, as a result of the hypoxia response element (HRE) embedded within its promoter sequence (23). 
Hypoxia represents a typical feature of almost all solid tumors. It is well known that solid tumors are characterized by increased growth and decreased cell death, causing significant oxygen deficiency (hypoxia) (23). In this regard, the expression and the enzymatic activity of LOX in tumors should be considered an important indicator of the transition from oxygen sufficiency to deficiency (23).

Active LOX molecules were most often associated with metastasis molecular mechanisms (24). In the context of metastasis EMT is a very important player, being involved in cells intravasation into the circulation, cells travel to the distant site, extravasation, and growth at this distant site (25). In order to understand the complex mechanism of each step of metastasis it is necessary a deep and thorough analysis of the extracellular matrix interactions at the cellular and molecular levels site (25). The LOX family members seem to play extremely important roles in these extracellular matrix interactions. These enzymes have multiple iso-

\section{BIBLIOGRAFIE}

1. Kaplan RN, Riba RD, Zacharoulis S, Bramley AH, Vincent L, Costa C et al. VEGFR1-positive haematopoietic bone marrow progenitors initiate the pre-metastatic niche. Nature. 2005 Dec 8;438(7069):820-7.

2. Erler JT, Bennewith KL, Cox TR, Lang G, Bird D, Koong A, Le QT, Giaccia AJ. Hypoxia-induced lysyl oxidase is a critical mediator of bone marrow cell recruitmentto form the premetastatic niche. Cancer Cell. 2009 Jan 6;15(1):35-44.

3. Hiratsuka S, Watanabe A, Aburatani H, Maru Y. Tumour-mediated upre-gulation of chemoattractants and recruitment of myeloid cells predeter-mines lung metastasis. Nat Cell Biol 2006;8:1369-75.

4. Harris ED. Copper-induced activation of aortic lysyl oxidase in vivo. Proc. Natl. Acad. Sci. USA 1976, 73, 371-374.

5. Siegel RC, Pinnell SR, Martin, GR. Cross-linking of collagen and elastin - Properties of lysyl oxidase. Biochemistry 1970, 9, 4486-4492.

6. Bove X, Ruiz-Trillo I, Rodriguez-Pascual F. Origin and evolution of lysyl oxidases. Sci Rep 2015;5:10568.

7. Selye H. Lathyrism. Revue canadienne de biologie/editee par l'Universite de Montreal. 1957; 16:1-82.

8. Tang SS, Trackman PC, Kagan HM. Reaction of aortic lysyl oxidase with beta-aminopropionitrile. The Journal of Biological Chemistry. 1983; 258:4331-4338.

9. Tang SS, Chichester CO, Kagan HM. Comparative sensitivities of purified preparations of lysyl oxidase and other amine oxidases to active site-directed enzyme inhibitors. Connective Tissue Research. 1989; 19:93-103.

10. Uzel MI, Scott IC, Babakhanlou-Chase H, Palamakumbura AH, Pappano WN, Hong HH, Greenspan DS, Trackman PC. Multiple bone morphogenetic protein 1-related mammalian metalloproteinases process pro-lysyl oxidase at the correct physiological site and control lysyl oxidase activation in mouse embryo fibroblast cultures. The Journal of Biological Chemistry. 2001; 276:22537-22543.

11. Peng L, Ran YL, Hu H, Yu L, Liu Q, Zhou Z, Sun YM, Sun LC, Pan J, Sun LX et al. Secreted LOXL2 is a novel therapeutic target that promotes gastric cancer metastasis via the Src/FAK pathway. Carcinogenesis 2009, 30, 1660-1669.

12. Mäki JM, Räsänen J, Tikkanen $H$, Sormunen R, Mäkikallio K, Kivirikko $\mathrm{KI}$, Soininen R. Inactivation of the lysyl oxidase gene Lox leads to aortic aneurysms cardiovascular dysfunction, and perinatal death in mice. Circulation 2002;106:2503-9. forms and different domains with opposing functions, which are incompletely understood yet site (25).

\section{CONCLUSIONS}

Elucidating the deep molecular mechanisms of the early events associated with solid tumor metastasis, including the discovery and characterization of the key effectors of this process, represent essential tasks for the development of efficient targeted cancer therapies. Further studies are required in order to elucidate the intimate mechanisms by which LOX molecules play their roles on the metastasis stage. Moreover, future research is needed to confirm whether simple LOX blocking or its downstream actions control could be regarded as target of preventive treatment in patients at a higher risk of metastasis. This is how the portrait of the future biomarker of LOX is becoming more and more outlined.

13. HM, Trackman PC. Properties and function of lysyl oxidase. Am J Respir Cell Mol Biol 1991;5:206-10.

14. Smith-Mungo L, Kagan H. Lysyl oxidase: properties, regulation and mul-tiple functions in biology. Matrix Biol 1998;16:387-98.

15. Pornprasertsuk S, Duarte WR, Mochida Y, Yamauchi M. Lysyl hydroxylase-2b directs collagen cross-linking pathways in MC3T3-E1 cells. Journal of Bone and Mineral Research: The Official Journal of the American Society for Bone and Mineral Research. 2004; 19:1349-1355.

16. Saito M, Marumo K. Effects of Collagen Crosslinking on Bone Material Properties in Health and Disease. Calcif Tissue Int. 2015; 97:242-261.

17. Heikkinen J, Risteli M, Wang C, Latvala J, Rossi M, Valtavaara M, Myllyla R. Lysyl hydroxylase 3 is a multifunctional protein possessing collagen glucosyltransferase activity. The Journal of Biological Chemistry. 2000; 275:36158-36163.

18. Giampuzzi M, Oleggini R, Albanese C, Pestell R, di Donato A. Beta-catenin signaling and regulation of cyclin D1 promoter in NRK-49F cells transformed by down-regulation of the tumor suppressor lysyl oxidase. Biochim. Biophys. Acta 2005, 1745, 370-381.

19. Oleggini R, Gastaldo N, di Donato A. Regulation of elastin promoter by lysyl oxidase and growth factors: Cross control of lysyl oxidase on TGF-1 effects. Matrix Biol. 2007, 26, 494-505.

20. Alsulaiman M, Bais MV, Trackman PC. Lysyl oxidase propeptide stimulates osteoblast and osteoclast differentiation and enhances PC3 and DU145 prostate cancer cell effects on bone in vivo. J. Cell Commun. Signal. 2016, 10, 17-31.

21. Myers BA, Dubick MA, Gerriets JE, Reiser KM, Last JA, Rucker RB. Lung collagen and elastin after ozone exposure in vitamin B-6-deficient rats. Toxicol. Lett. 1986, 30, 55-61.

22. Nagan N, Kagan HM. Modulation of lysyl oxidase activity toward peptidyl lysine by vicinal dicarboxylic amino acid residues. Implications for collagen cross-linking. The Journal of biological chemistry. 1994; 269:22366-22371.

23. Yang X, Li S, Li W, Chen J, Xiao X, Wang Y, Yan G, Chen L. Inactivation of lysyl oxidase by aminopropionitrile inhibits hypoxia-induced invasion and migration of cervical cancer cells. Oncol. Rep. 2013, 29, 541-548.

24. Barker HE, Cox TR, Erler JT. The rationale for targeting the LOX family in cancer. Nature reviews. Cancer. 2012; 12:540-552.

25. Valastyan S, Weinberg RA. Tumor metastasis: Molecular insights and evolving paradigms. Cell. 2011; 147:275-292. 